\title{
A two-level parallelization method for distributed hydrological models
}

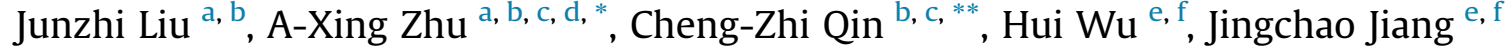 \\ a Key Laboratory of Virtual Geographic Environment, Ministry of Education, Nanjing Normal University, 1 Wenyuan Road, Nanjing, Jiangsu 210023, China \\ ${ }^{\mathrm{b}}$ Jiangsu Center for Collaborative Innovation in Geographical Information Resource Development and Application, 1 Wenyuan Road, Nanjing, Jiangsu \\ 210023, China \\ c State Key Lab of Resources and Environmental Information System, Institute of Geographic Sciences and Natural Resources Research, Chinese Academy of \\ Sciences, Beijing, 100101, China \\ d Department of Geography, University of Wisconsin-Madison, Madison, WI 53706, USA

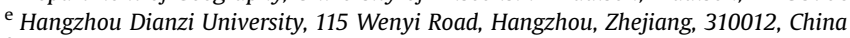 \\ ${ }^{\mathrm{f}}$ Smart City Research Center of Zhejiang Province, 115 Wenyi Road, Hangzhou, Zhejiang, 310012, China
}

\section{A R T I C L E I N F O}

\section{Article history:}

Received 29 October 2015

Received in revised form 28 February 2016

Accepted 28 February 2016

Available online 9 March 2016

\section{Keywords:}

Distributed hydrological model

Two-level parallelization

Multi-core cluster

Sub-basin

Basic simulation unit

\begin{abstract}
A B S T R A C T
This paper proposes a scalable two-level parallelization method for distributed hydrological models that can use parallelizability at both the sub-basin level and the basic simulation-unit level (e.g., grid cell) simultaneously. This approach first uses the message-passing programming model to dispatch parallel tasks at the sub-basin level to different nodes with multi-core CPUs in the cluster. Each node is responsible for some of the sub-basins. Parallel tasks for each sub-basin at the basic simulation-unit level are then dispatched to multiple cores within each node using the shared-memory programming model. A grid-based distributed hydrological model was parallelized to demonstrate the performance of the proposed method, which was tested in different scenarios (e.g., different data volume, different numbers of sub-basins). Results show that the proposed two-level parallelization method had better scalability than the parallel computation at sub-basin level alone, and the parallel performance increased with data volume and the number of sub-basins.
\end{abstract}

๑) 2016 Elsevier Ltd. All rights reserved.

\author{
Software availability \\ Program title: TwoLevelParHydroModel. \\ Description: Two-level parallel computing for distributed \\ hydrological modeling. \\ Developer: Dr. Junzhi Liu and Prof. A-Xing Zhu. \\ Platform: Linux. \\ Source language: $\mathrm{C}++$
}

\footnotetext{
* Corresponding author. Key Laboratory of Virtual Geographic Environment, Ministry of Education, Nanjing Normal University, 1 Wenyuan Road, Nanjing, Jiangsu 210023, China.

** Corresponding author. State Key Lab of Resources and Environmental Information System, Institute of Geographic Sciences and Natural Resources Research, Chinese Academy of Sciences, Beijing, 100101, China.

E-mail addresses: axing@njnu.edu.cn (A.-X. Zhu), qincz@lreis.ac.cn (C.-Z. Qin).
}

Cost: Free.

Availability: Contact the developer.

\section{Introduction}

There are several emerging trends in distributed hydrological modeling, which plays an important role in watershed research and management (Borah and Bera, 2004). First, high spatial resolutions are needed to characterize the detailed spatial distribution of hydrological variables such as soil moisture and soil erosion (Rojas et al., 2008; Chen et al., 2013). Second, long-term simulations are needed to assess the impacts of land-use change and climate change on watershed sustainable development (Bhaduri et al., 2001; Barnett et al., 2005). Third, integrated simulations coupling multiple geographic processes are needed in order to evaluate the effects of watershed management practices comprehensively (Zoltay et al., 2010). To conduct such distributed hydrological 
modeling over long periods, a large amount of computation are required. This makes the parallelization of distributed hydrological models an inevitable choice (Vivoni et al., 2011).

In distributed hydrological modeling, watersheds are divided into different types of simulation units, such as sub-basins, grid cells and hydrological response units (Dehotin and Braud, 2008). It is a common way to dispatch computing tasks of different simulation units to multiple processors for parallel computing ( $\mathrm{Li}$ et al., 2011). For example, parallel-computing methods at different levels (e.g., sub-basin or grid cell) using different types of computer hardware (e.g., cluster or multi-core CPU [Central Processing Unit]) have been proposed and proven to be effective with speedup ratios ranging from 2 to 80 (Kollet and Maxwell, 2006; Vivoni et al., 2011; Wang et al., 2011; Ran et al., 2013; Yalew et al., 2013; Burger et al., 2014; Liu et al., 2014).

However, the scalability of parallel computing using the parallelizability among simulation units at a single level is usually limited. For example, there exist maximum speedup ratios (MSRs) for parallel computing at the sub-basin level (Liu et al., 2013). When the number of processors exceeds certain thresholds, the speedup ratio will not keep increasing due to the load imbalance caused by limited numbers of sub-basins and the dependences among them (Wang et al., 2012; Liu et al., 2013). Parallel computing at the gridcell level also has scalability problems mainly due to communication overhead (Liu et al., 2014). How to address the scalability problem and improve the efficiency of parallel computing for distributed hydrological models has become an important research topic.

This paper proposes a two-level parallelization method for distributed hydrological models by simultaneously utilizing the parallelizability at both the sub-basin and the basic simulation unit levels, and in this way improve parallel-computing scalability. Section 2 describes the basic concept of the two-level parallelization method. Section 3 describes the implementation of the method, taking a grid-based distributed hydrological model as an example. Section 4 validates the effectiveness of the proposed method through a case study. Section 5 concludes and discusses future research directions.

\section{Basic concept of the two-level parallelization method}

For distributed hydrological modeling, a watershed can be divided into spatial units of different levels. The spatial units from top to bottom are usually sub-basins, hillslopes and basic simulation units such as grid cells and hydrological response units (Band et al., 2000). Parallel computing for distributed hydrological modeling can be conducted at different levels. The granularity of parallel tasks, which refers to the number of computations for each parallel task and the communication overhead among parallel tasks, are different for parallel computing at different levels. Parallel computing at the sub-basin and hillslope levels is coarse grained, with a relatively low number of parallel tasks and low communication overhead, and it is suitable to run on computer clusters. Parallel computing at this level has good scalability with respect to hardware, but suffers from limited parallelizability caused by the limited number of parallel tasks and the dependences among them (Wang et al., 2012). In contrast, parallel computing at the basic simulation-unit level (e.g., grid-cell level) is fine-grained, with a relatively large number of parallel tasks but also with high communication overhead. Although there is good parallelizability at this level, it requires an extensive level of communication and so need to run on shared-memory hardware. The hardware environment is usually the limiting factor for parallel computing at this level (Liu et al., 2014). The coarse- and finegrained parallel computing methods described above are complementary on the aspects of parallelizability and hardware. Combining them to conduct two-level parallel computing may lead to better parallel-computing performance (Zhao et al., 2013).

Because parallel computing at the sub-basin level is suitable to run on computer clusters, and at the basic simulation-unit level it is suitable to run on shared-memory hardware, multi-core cluster (i.e. a cluster having nodes with multi-core CPUs), is selected as the hardware platform for the two-level parallel computation in this paper. With the development of multi-core CPUs, most current computer clusters are multi-core clusters, which made the proposed method in this study widely applicable. The architecture of the two-level approach is as follows: first, parallel computing at the sub-basin level is conducted among multiple nodes in a computer cluster using the message-passing programming model, and then parallel computing at the basic simulation-unit level is conducted among multi-cores within each node using the shared-memory programming model (Fig. 1). It is worth noting that although a job management systems like PBS (Portable Batch System) could also be used to conduct the coarse-grained parallel computation, the intense interaction among parallel tasks at the sub-basin level requires a flexible communication mechanism and makes the message-passing parallel computation (e.g. using MPI (Message Passing Interface)) a necessary choice.

\section{Implementation of the two-level parallelization method}

The two-level parallelization method is a combination of parallel computing at the sub-basin level and at the basic simulationunit level. To illustrate the implementation of this method, a gridbased distributed hydrological model was parallelized as an example.

\subsection{Description of a grid-based distributed hydrological model}

The grid-based distributed hydrological model we used was developed by Liu et al. (2014), which integrates several existing methods to conduct event-based hydrological simulation in semiarid watersheds. The hydrological processes simulated in this model include interception, infiltration, surface depression, overland-flow routing and channel-flow routing. The interflowand groundwater-flow processes were omitted because runoff generation is dominated by infiltration excess overland flow in semi-arid areas.

The infiltration process was simulated using a quadratic approximation of the Green-Ampt method (Li et al., 1975). The interception and depression processes were simulated using methods in the Wetspa model (Liu and De Smedt, 2005). For simulation of the interception process, the fill-and-spill mechanism was used and the maximum interception storage was calculated using a statistical equation containing leaf area index, vegetal species, and date (Liu and De Smedt, 2004). To simulate the depression process, depression and overland flow were allowed to occur simultaneously even if excess rainfall was less than the depression storage, which was estimated by an empirical equation suggested by Linsley et al. (1975).

Both overland-flow routing and channel-flow routing were performed sequentially from upstream to downstream according to the single-flow direction defined by the D8 method (O'Callaghan and Mark, 1984). Surface flow was simulated by a onedimensional kinematic wave model combined with the Manning's equation (Chow et al., 1988). The equation for surface-water depth is 
Parallel computing at the sub-basin level using the message-passing model

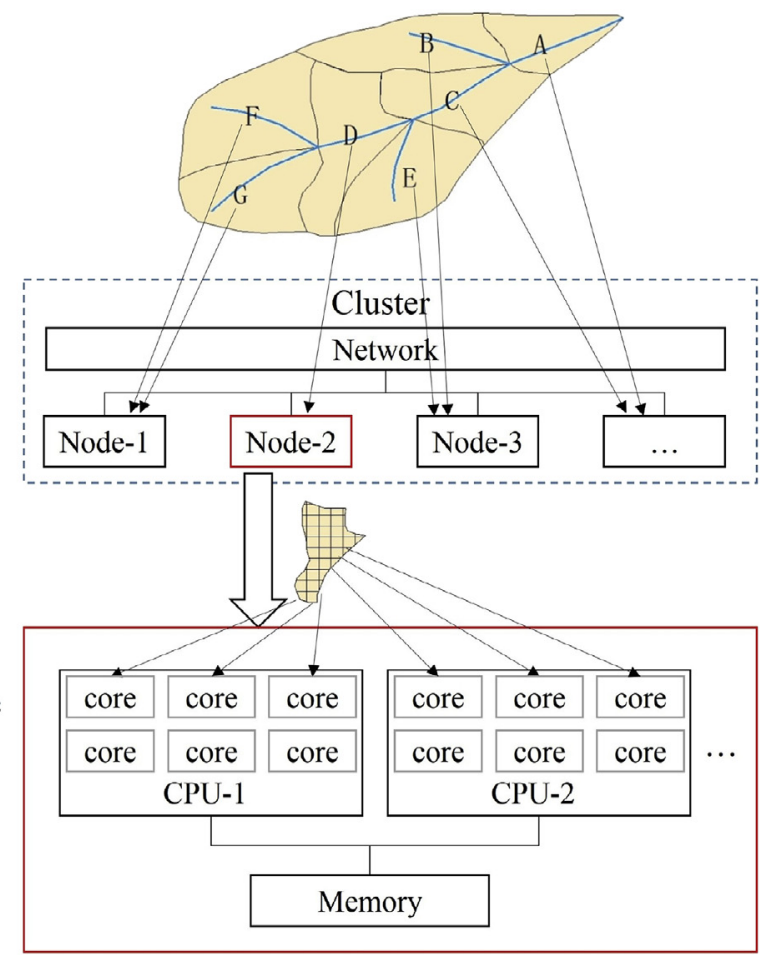

Fig. 1. Architecture of the two-level parallel-computing approach.

$\frac{\partial h}{\partial t}+\frac{1}{n} \sqrt{S_{0}} \frac{\partial\left(h^{5 / 3}\right)}{\partial \mathrm{x}}=\mathrm{i}-\mathrm{f}$

where $h$ is the depth of water on the surface (m); $t$ is the time (s); $n$ is the Manning coefficient; $S_{0}$ is the bed slope $(\mathrm{m} / \mathrm{m}) ; x$ is the length of the slope $(\mathrm{m}) ; i$ is the rainfall intensity $(\mathrm{m} / \mathrm{s}) ; f$ is the infiltration rate $(\mathrm{m} / \mathrm{s})$. This equation was solved using a combination of the 4point implicit finite difference and Newton's iteration method.

\subsection{Parallel computing at the sub-basin level}

\subsubsection{Assignment of parallel tasks}

Parallel computing at the sub-basin level was conducted using message-passing programming models, with parallel tasks dispatched to different nodes in a cluster to run in parallel. Process is the basic scheduling unit for message-passing parallel computing, and one or several processes run on a node. For the assignment of parallel tasks, the amount of computation in each process should be distributed as evenly as possible, while the amount of communication should be as minimal as possible. There is a tradeoff between these two goals, and this problem has been proved to be NP-hard (Garey et al., 1976).

In this paper, sub-basins were treated as a graph (Fig. 2) and the widely used METIS graph-partitioning algorithm (Karypis and Kumar, 1998) was used for the assignment of parallel tasks. Each sub-basin was represented as a node in the graph. The weight of the graph was defined as the number of grid cells in this sub-basin, because generally a higher number of grid cells requires more execution time. The dependence relationship between two subbasins was represented as an arc in the graph. For the distributed hydrological model used in this paper, there are only dependencies between upstream and downstream sub-basins due to channel routing.

\subsubsection{The scheduling of parallel tasks}

For the hydrological model used in this paper, channel routing causes dependent relationships between sub-basins, and the hillslope processes between sub-basins are independent. From the perspective of geographic space, for headwater sub-basins, both hillslope and channel-routing processes are independent; for nonheadwater sub-basins, hillslope processes are independent, but the channel-routing process depends on its upstream sub-basins.

In light of these factors, the computational tasks at the sub-basin level were divided into three types (Fig. 3): (1) the computational tasks of hydrological processes (including both hillslope and channel-routing processes) in headwater sub-basins; (2) the computational tasks of hillslope processes in non-headwater subbasins; (3) the computational tasks of the channel routing process in non-headwater sub-basins. The first two types of computational tasks are independent of each other, so they can be conducted in parallel directly. For the third type of computational task, there are dependent relationships between upstream and downstream subbasins, so computational tasks should be conducted following a sequence from upstream to downstream. In other words, the computational tasks of a downstream sub-basin cannot be conducted until the computations for all of its upstream sub-basins have been finished. If adjacent upstream and downstream subbasins are not in the same group of parallel tasks, communication is needed.

Based on the classification of computational tasks described above, a master-slave task scheduling framework (Li et al., 2011; Wang et al., 2011, 2013) was proposed to conduct parallel computing, which consists of three components: a master process, several slave processes and a hydrological database (Fig. 4). The master process allocates tasks and manages the communication among slave processes, the slave processes conduct the actual hydrological simulation tasks, and the hydrological database is used to store input and output data. Details of these three components are described below. 

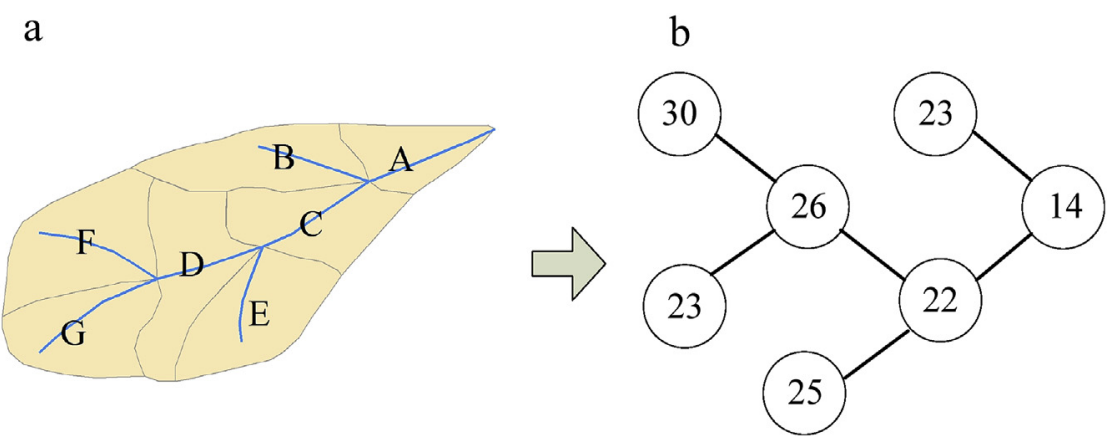

Fig. 2. (a) Sub-basins and (b) their graph representation. Numbers in graph nodes are node weights.

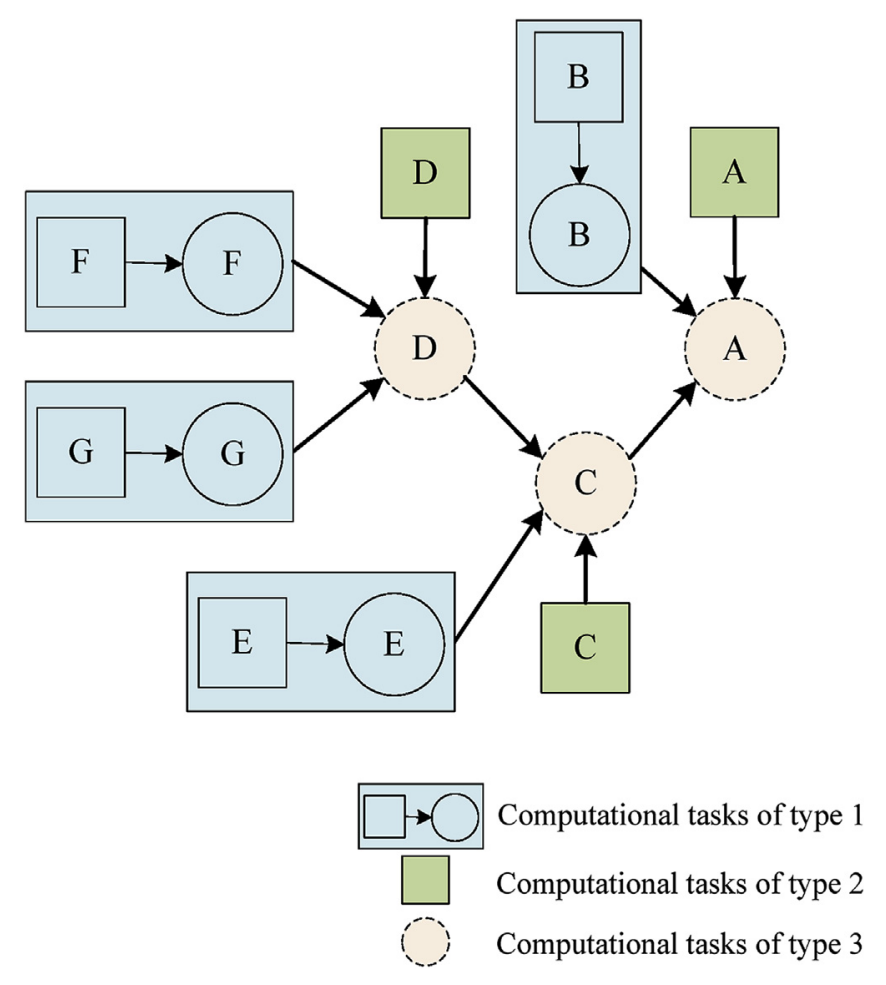

Fig. 3. Classification of parallel tasks for distributed hydrological modeling at subbasin level. Type 1: computational tasks of hydrological processes (including both hillslope and channel-routing processes) in headwater sub-basins; type 2: computational tasks of hillslope processes in non-headwater sub-basins; type 3: computational tasks of the channel-routing process in non-headwater sub-basins. Rectangles represent hillslope processes; circles represent channel-routing processes.

3.2.2.1. Hydrological database. The hydrological database in the above parallel-computing framework stores both the input and output data of the model. The input data include climate data (e.g., precipitation and temperature), geographic data (e.g., Digital Elevation Model [DEM], land-use map and soil-type map) and spatial parameters for distributed hydrological modeling (e.g., saturated hydraulic conductivity, interception storage). The output data include time-series output at the outlet (e.g., discharge) and spatiotemporal distribution of hydrological variables (e.g., soil moisture). In order to read and save data effectively at the sub-basin level, all spatial input data were decomposed into small parts according to sub-basin boundaries and stored in the database as binary types.

3.2.2.2. Master process. There are two stages in the execution of the master process: the task-allocation stage and the message-listening stage. During the first stage, the master process allocates computing tasks to slave processes according to the task assignment solution obtained from the METIS algorithm. As there are close data connections between the hillslope processes and the channel-routing process in the same sub-basin (e.g., lateral flow from hillslope to channel), the computational tasks of one subbasin are dispatched as a whole from the master process to slave processes. Once the task allocation is complete, the master process enters the message-listening stage, in which it continues to receive and respond to messages. There are three cases that vary according to the type of messages received from slave processes:

(1) If the message contains the routing-calculation results of a sub-basin, the master process will record the results in memory and check whether they are needed by other slave processes. If they are needed as input, the master process will transfer them to that slave process.

(2) If the message is a request for upstream inflows of a subbasin, the master process will check whether the upstream inflow data are already available in memory. If so, it will send these inflow data back to the slave process; otherwise, it will add the request to the waiting list, so that the requested data will be sent as soon as they are available.

(3) If the message contains the information that all tasks have been completed by the slave processes, the master process will quit.

3.2.2.3. Slave process. The slave processes are in charge of conducting actual calculations of hydrological simulations. After receiving computing tasks from the master process, the slave processes read input data and parameters from the hydrological database. The calculations of hydrological simulations, which contain time loops, then begin. During the calculation, the execution sequence of computational tasks was determined according to the type of task (as shown in Fig. 3). The computational tasks of hydrological processes (including both hillslope and channelrouting processes) in headwater sub-basins (type 1) and those of hillslope processes in non-headwater sub-basins (type 2) are independent among sub-basins, so they were conducted first at each time step. The computational tasks of the channel-routing process in non-headwater sub-basins (type 3) depend on calculation results from the first two types of tasks, so they will not be conducted until the type 1 and type 2 tasks are finished.

The channel-routing results of upstream sub-basins are needed for the channel-routing calculation of their downstream subbasins. So when a sub-basin's type 1 and 3 computational tasks, which contain channel-routing processes, are complete, the slave 


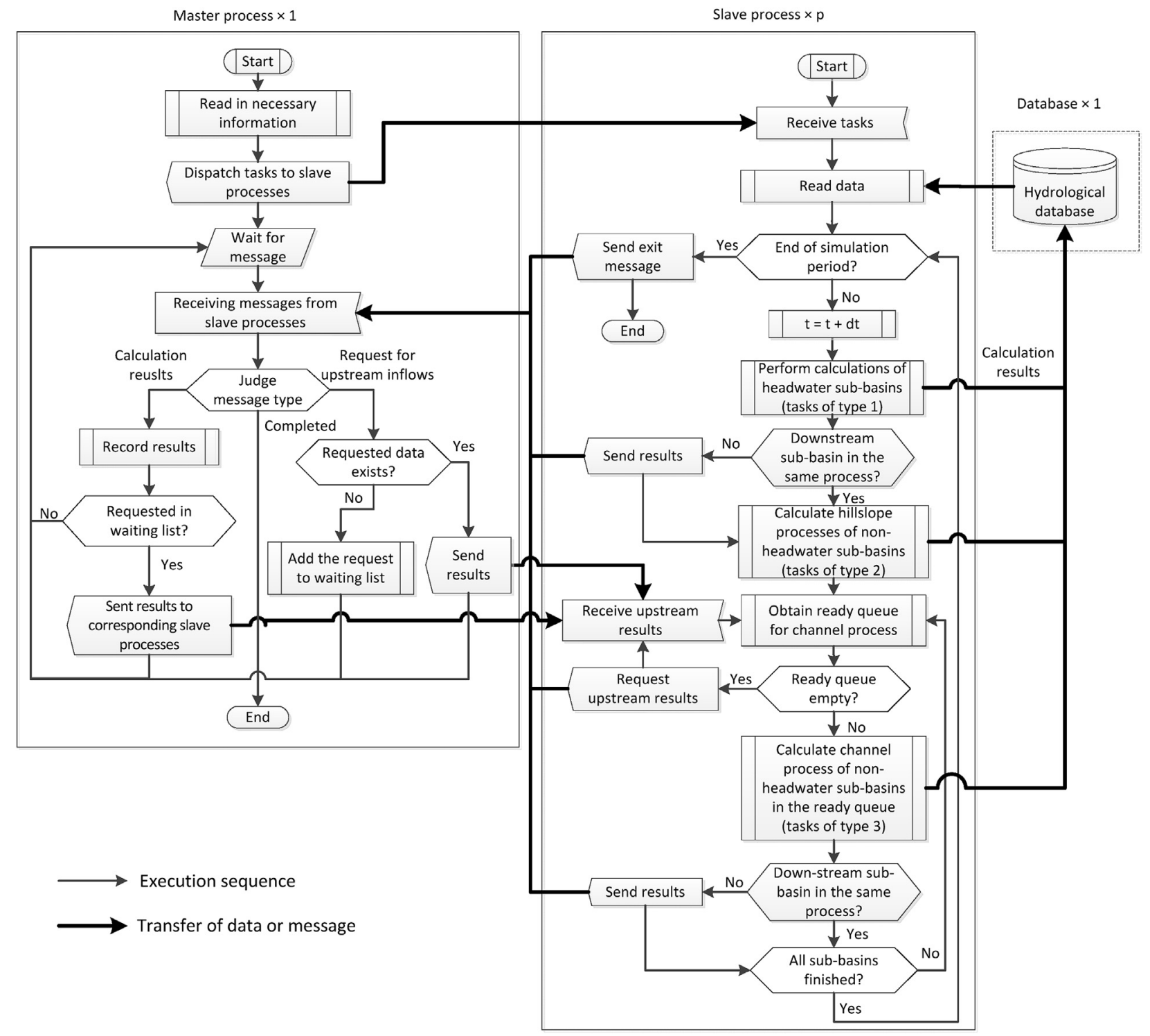

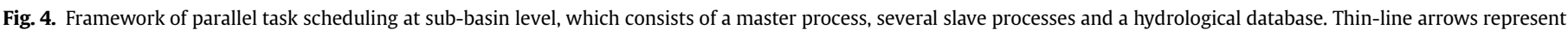
execution sequences and bold-line arrows represent transfers of message or data.

process will check whether the downstream sub-basin is in the same process. If so, the routing results of the sub-basin are saved in memory in order to be used by its downstream sub-basin; otherwise, the routing results are sent to the master process so that they can be transferred to the slave process that needs them.

For the type 3 computational tasks, only those sub-basins with available upstream inflows could be processed, and a queue was used to record these sub-basins. There is a loop to add processable sub-basins to the queue and then conduct the channel-routing calculations of sub-basins in the queue. If the queue becomes empty and there are still sub-basins to be processed, the slave process will send a request for upstream inflows to the master process and wait for responses. When the needed upstream inflow data from the master process are received, the loop will continue until all the sub-basins are processed.

\subsection{Parallel computing at the basic simulation-unit level}

The basic simulation units of the distributed hydrological model used in this paper are grid cells. At the grid-cell level, the layered parallel-computing approach proposed by Liu et al. (2014) was used. This approach was based on the shared-memory programming model, in which threads were the basic scheduling units. To conduct parallel computing, hydrological processes are divided into two types: runoff generation processes and overland-flow routing processes. For runoff generation processes, calculations for different grid cells are independent; parallel computing can therefore be conducted simply by dividing grid cells into equal parts.

For the overland-flow routing process, there are sequential dependences among upstream and downstream grid cells, so in order to conduct parallel computing, this approach divided grid cells into layers according to flow topology (Fig. 5). The single-flow direction (D8) method (O'Callaghan and Mark, 1984) was used to define the flow topology. The outlet cell is labeled layer 1 and the units draining directly to cells in layer 1 are defined as layer 2 . The cells draining directly to cells in layer $n$ are defined as layer $n+1$ and so on until the most upstream cells are reached (Wang et al., 2004). In 
a

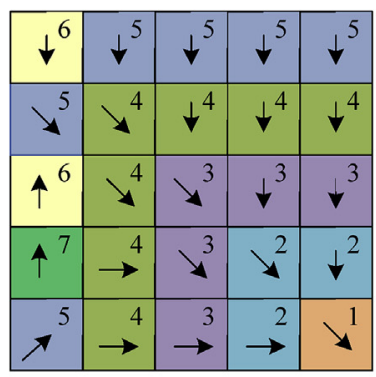

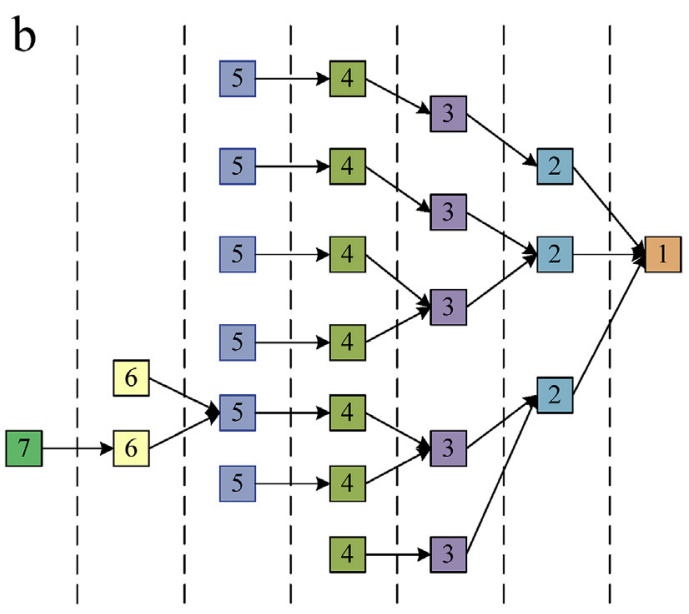

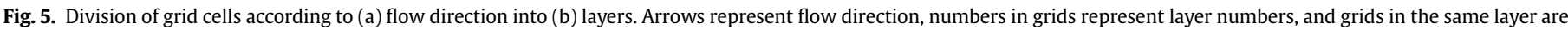
filled with the same color.

such a way, there are no upstream or downstream relationships among grid cells within each layer. Therefore, the calculations on grid cells in the same layer are independent and can be conducted in parallel.

\subsection{Programming implementation}

The proposed two-level parallelization method was implemented using standard $\mathrm{C}++$ programming language. For the message-passing parallel computing at the sub-basin level, the MPI communication protocol was used. MPI is the de facto standard for message-passing parallel programming, and provides a set of send and receive functions that allow for communication among different processes. For shared-memory parallel computing at the grid-cell level, the OpenMP (Open Multi-Processing) application programming interface was adopted. OpenMP is based on the fork/ join programming model and parallel computation was conducted by embedding OpenMP compiler directives in source code (Mattson et al., 2004).

\section{Case studies}

\subsection{Research design}

\subsubsection{Study area and dataset}

The Qingshuihe watershed, located in the Hebei province of China, was selected as the study area (Fig. 6). It has a drainage area of $6366 \mathrm{~km}^{2}$ and is located within $40^{\circ} 34^{\prime}-41^{\circ} 28^{\prime} \mathrm{N}$ and $114^{\circ} 43^{\prime}-115^{\circ} 36^{\prime} \mathrm{E}$. Elevation ranges from 781 to $2164 \mathrm{~m}$ above sea level, with an average basin slope of $13 \%$ derived from a $90 \times 90 \mathrm{~m}^{2}$ SRTM (Shuttle Radar Topography Mission) DEM. It has a sub-arid climate characterized by an average annual precipitation of $480 \mathrm{~mm}, 80 \%$ of which occurs between June and September in the form of intense storms.

Three datasets of different spatial resolutions $(30 \mathrm{~m}, 90 \mathrm{~m}$ and $270 \mathrm{~m}$ ) were used to test the performance of the proposed parallelization method (Table 1). The finer the spatial resolution is, the larger the data volume used in a simulation will be. In order determine the impact of data volume on parallel performance, the watershed was divided into the same number of sub-basins (i.e., 67 in this case study) for these three datasets to keep the communication costs among sub-basins roughly the same. And in order to determine the impact of the number of sub-basins on parallel performance, sub-basins of different numbers (i.e., 43, 67, and 89) were delineated for the $90 \mathrm{~m}$-resolution dataset. All experiments were performed using a 24-h storm with a 50-year recurrence interval at a 1-min time step. The default input parameters extracted from DEM, land-use map, and soil map, and from values described in the literature were used in the simulations. It is worth noting that the simulation results would change with the changes of spatial resolution, watershed subdivision and model parameters (Liang et al., 2004; Hessel, 2005; Tripathi et al., 2006). However, because the major focus of this study was to test the performance of parallel computation, the relationships between the simulation results and their affecting factors were not investigated.

\subsubsection{Hardware environment}

A computer cluster with 1 head node and 4 compute nodes was used as the hardware platform. These nodes were connected by 1Gigabit Ethernet. Each node had 2 Intel ${ }^{\circledR}$ Xeon E5645 CPUs and each CPU had 6 cores. There were 48 cores in all in the compute nodes of this cluster. The CentOS 6.0 Operating System, GCC 4.1.2 compiler and OpenMPI 1.3.3 were used.

\subsubsection{Experiment design}

The results of the serial and parallel simulations were confirmed as identical, and then three experiments were designed to test the parallel performance of the proposed two-level parallel-computing method. First, parallel computation was conducted under different combinations of processes and thread numbers to test the overall performance. The number of processes ranges from 1 to 48 and each process may contain 1, 2, 3 or 6 threads as long as the number of processes multiplied by the number of threads within each process was less than or equal to 48 , which was the total number of cores in compute nodes. The $90 \mathrm{~m}$-resultion dataset with 67 subbasins was used for the experiment. In order to investigate the impact of the number of sub-basins on parallel performance, twolevel parallel computation was then conducted using the $90 \mathrm{~m}$ resolution dataset with different numbers of sub-basins $(43,67$, and 89). Each process contained 2 threads. In order to study the impact of data volume on parallel performance, a two-tier parallel computation was finally conducted using datasets of different resolutions (i.e., $270 \mathrm{~m}, 90 \mathrm{~m}$ and $30 \mathrm{~m}$ ) with a fixed number of subbasins (i.e., 67). Each process also contained 2 threads. Each experiment was repeated three times to get an average computing time. The computing time here refers to the time for the actual simulation excluding the time for data input/output $(\mathrm{I} / \mathrm{O})$. 

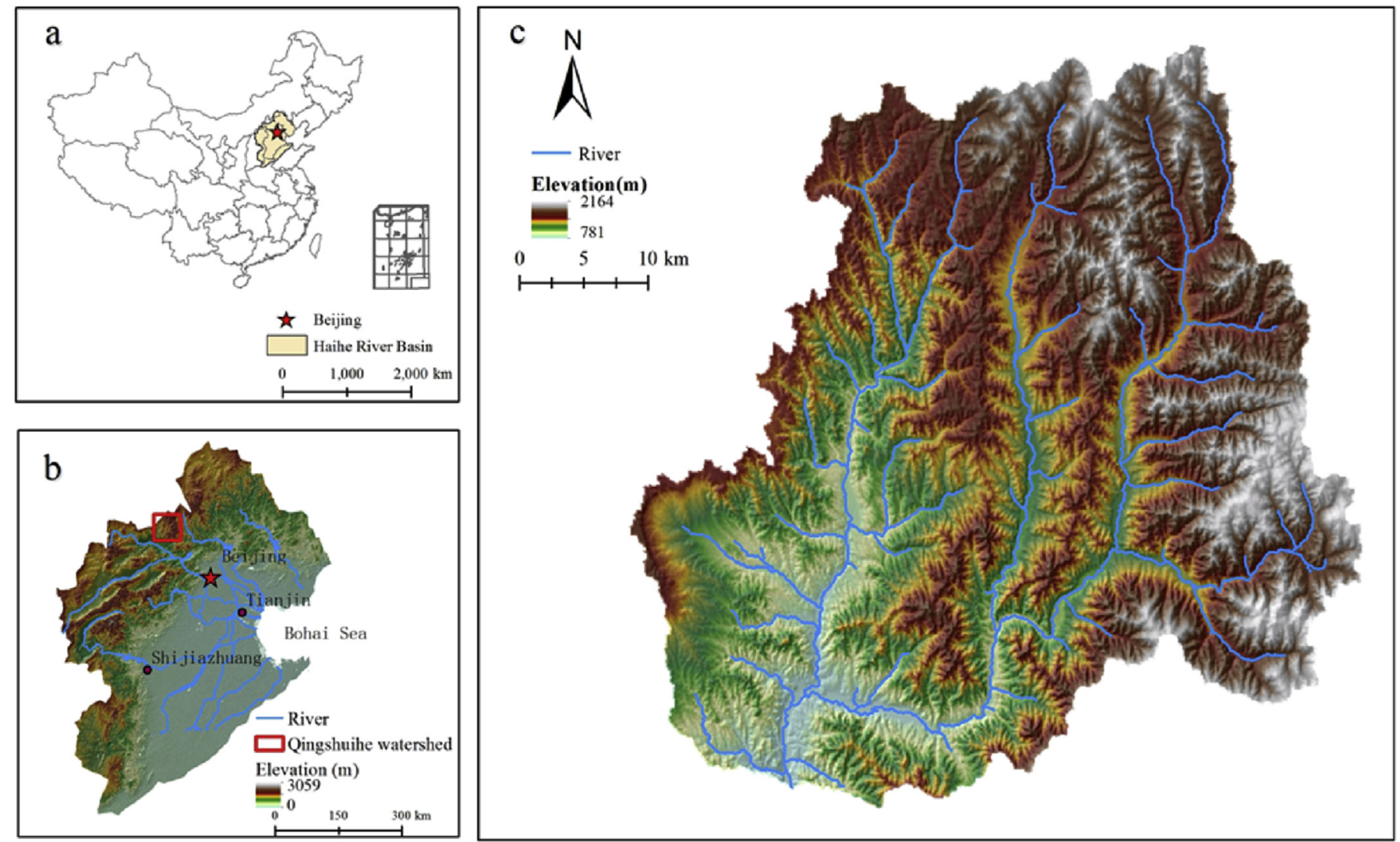

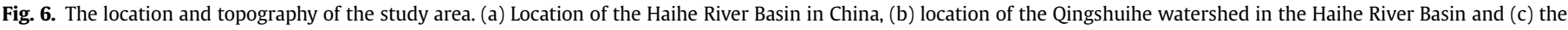
topography of the Qingshuihe watershed.

Table 1

Datasets of spatial resolutions and number of sub-basins.

\begin{tabular}{lll}
\hline Resolution $(\mathrm{m})$ & Number of grids & Number of sub-basins \\
\hline 270 & 29,850 & 67 \\
90 & 268,860 & $43,67,89$ \\
30 & $2,381,602$ & 67 \\
\hline
\end{tabular}

\subsubsection{Performance evaluation}

The speedup ratio and parallel efficiency were used to measure the performance of the proposed method. The speedup ratio is defined as the ratio between the serial computing time and the parallel computing time (Rauber and Rünger, 2010) and the equation is as follows:

$\mathrm{S}=\frac{T_{S}}{T_{p}}$

where $S$ is the speedup ratio; $T_{S}$ is the serial execution/computing time; $T_{p}$ is the parallel execution/computing time. Parallel efficiency is defined as the ratio between the speedup ratio and the number of cores used in the parallel computation (Rauber and Rünger, 2010) and the equation is as follows:

$\mathrm{E}=\frac{\mathrm{S}}{\mathrm{N}}$

where $E$ is the parallel efficiency; $N$ is the number of cores.

\subsection{Results and discussion}

\subsubsection{Overall performance}

Fig. 7 presents the execution time, speedup ratios and parallel efficiencies for the two-level parallel computation using different combinations of processes and thread numbers. A 90 m-resultion dataset with 67 sub-basins was used for the experiment. The number of cores in Fig. 7 refers to the number of cores used in parallel computation, which was equal to the number of processes multiplied by the number of threads within each process. When there was one thread within each process, only a one-tier parallel computation at the sub-basin level was conducted. When there was more than one thread within each process, a two-level parallel computation was conducted.

In general, the execution time decreased and the speedup ratios increased with the number of cores used in parallel computation for all the experiments. However, for the parallel computation at sub-basin level alone (i.e., the experiment with 1 thread per process), the speedup ratios reached a plateau when the number of cores exceeded 24. For the two-level parallel computation, the scalability was better than the parallel computation at sub-basin level alone. When the number of cores exceeded 24, the speedup ratios of two-level parallel computation were larger than those of the parallel computation at sub-basin level alone. This is because the parallel efficiencies at the sub-basin level were limited by the load imbalance of computing tasks and communication overhead at the sub-basin level. The more the number of tasks that were divided at sub-basin level, the worse the load imbalance and communication overhead problem will become, thus the parallel computation is less efficient. By utilizing parallelizability at a finer 

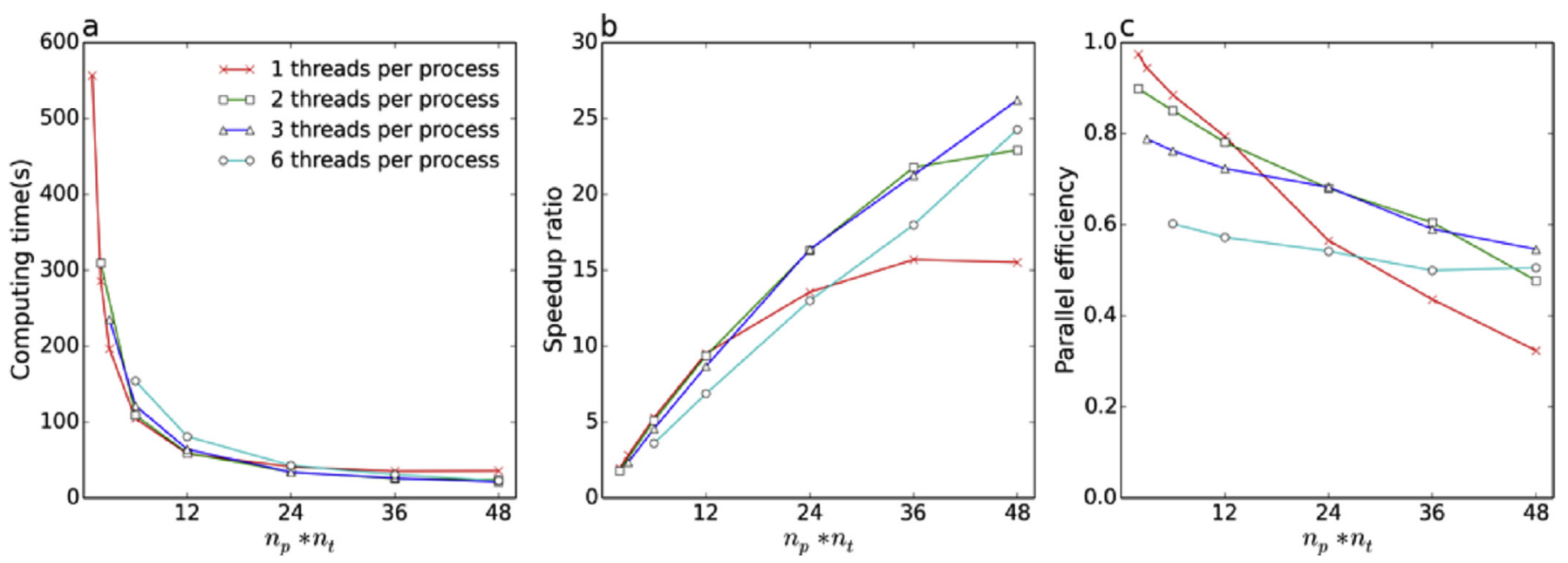

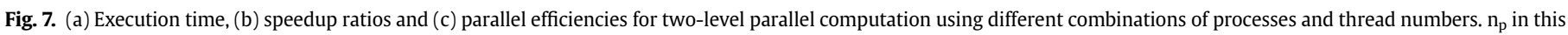
figure means the number of cores and $n_{t}$ means the number of threads within each process.

level (the grid-cell level in this case) as well as that at the sub-basin level, two-level parallel computation required a smaller number of parallel tasks at the sub-basin level to make use of a given number of cores compared to parallel computation at the sub-basin level alone. In this way, the load imbalance and communication overhead problem could be relieved.

The speedup ratios of the two-tier parallel computation would reach a plateau when the number of cores used in parallel computation exceeded a larger threshold. This threshold, on one hand, depended on parallelizability at the sub-basin level. The shape of the watershed and the number of sub-basins affected parallel performance (Liu et al., 2013). On the other hand, this threshold depended on parallelizability at the grid-cell level. If the scalability of parallel computation at the grid-cell level was good enough, the higher the number of threads within each process, the better the scalability of the two-level parallel computation. Although the threshold still existed, the scalability of the two-level parallel computation was effectively improved compared to that of the parallel computation at sub-basin level alone.

\subsubsection{Impact of the number of sub-basins on parallel performance}

In order to investigate the impact of the number of sub-basins on parallel performance, a two-level parallel computation was conducted using the $90 \mathrm{~m}$-resolution dataset with different numbers of sub-basins $(43,67$, and 89$)$. The results are shown in Fig. 8. The parallel computation with a greater number of subbasins had better parallel performance when the number of cores exceeded 36 . This could be attributed to the increasing parallelizability at the sub-basin level with the increase in the number of sub-basins, which then led to better scalability of the parallel computation. However, it should be kept in mind that sub-basin delineation should not be driven by the needs of parallel computation but should be determined by the needs of hydrological studies in certain scenarios. The number of sub-basins cannot be increased arbitrarily in order to get better parallel performance.

\subsubsection{Impact of data volume on parallel performance}

The performance of two-tier parallel computation with different volumes of data was tested using datasets of different resolutions (i.e., $270 \mathrm{~m}, 90 \mathrm{~m}$ and $30 \mathrm{~m}$ ) with a fixed number of sub-basins (67) and the results are shown in Fig. 9. The figure shows that data volume had a direct influence on parallelization performance. The larger the dataset, the higher the speedup for a given number of cores. This is because the time spent on actual calculation increased with data volume, which made the communication overhead decrease at both the sub-basin level and the grid-cell level, leading to better parallelization performance.

\section{Conclusions}

This paper proposed a two-level parallelization method for distributed hydrological models, which can utilize parallelizability at both the sub-basin level and the basic simulation-unit level (e.g., grid-cell level) simultaneously using a multi-core cluster as the hardware platform. The basic concept of the proposed two-level approach is to first dispatch parallel tasks at the sub-basin level to multiple nodes in a computer cluster in order to conduct parallel computation using the message-passing programming model. Parallel tasks at the basic simulation-unit level are then dispatched to multi-cores within each node to conduct parallel computation using the shared-memory programming model. To illustrate the method, a grid-based distributed hydrological model was parallelized as an example. The performance of the parallelized model was tested in different scenarios. The results showed that the proposed method had better performance than parallel computation at the sub-basin level alone, and the parallel scalability increased with data volume and the number of sub-basins.

Due to the lack of high-resolution meteorological and discharge data in our study area, we just used designed storm instead of meteorological observation data to drive the model and test its parallel performance. In the future, we will collect more data and conduct real-world assessment of the parallelized distributed hydrological model. In addition, in the proposed two-level parallelization method, the number of cores used in a specific compute node equaled the number of processes multiplied by the number of threads within each process. Before submitting an execution command to a multi-core cluster, users need to coordinate the number of processes running in each node and the number of threads within each process to avoid overburdening some compute nodes. This introduces some inconvenience for users. The next step would be to study the ease of software use and to utilize the multilevel parallelizability in hydrological models using only the message-passing programming model, which is the most widely used model for massive parallel computing. 

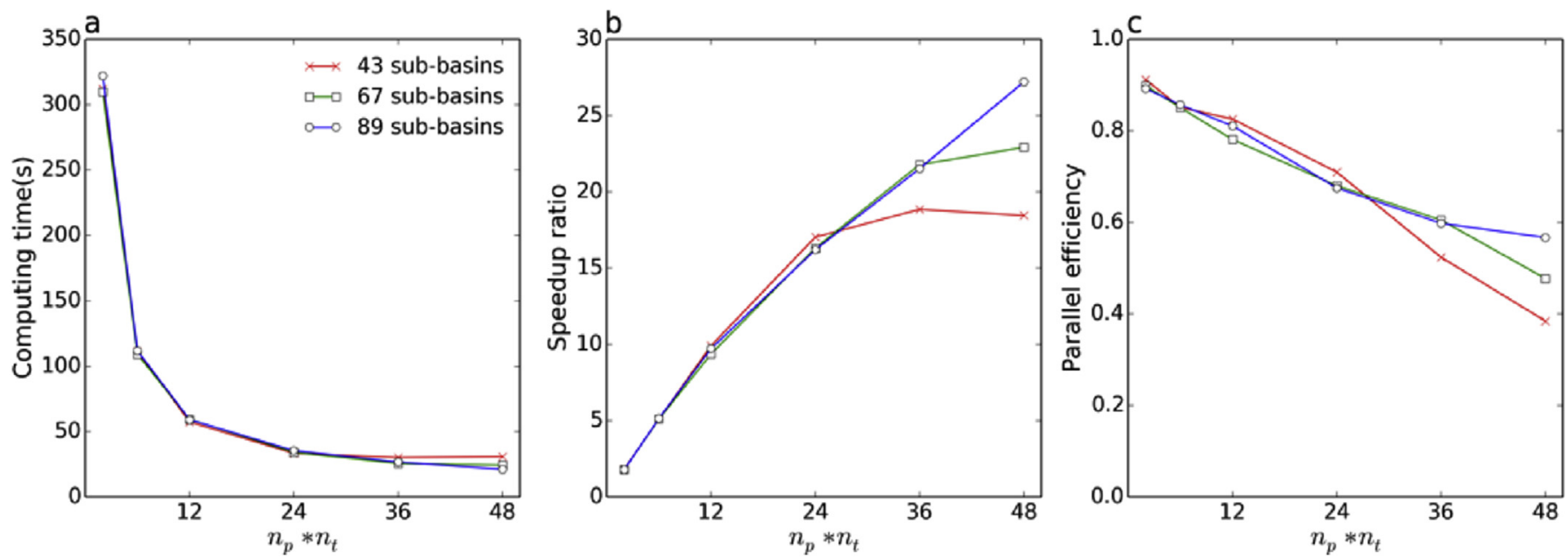

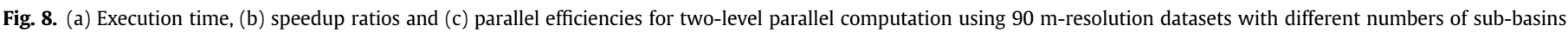
(i.e., 45,67 , and 89 ). $n_{p}$ in this figure means the number of cores and $n_{t}$ means the number of threads within each process.
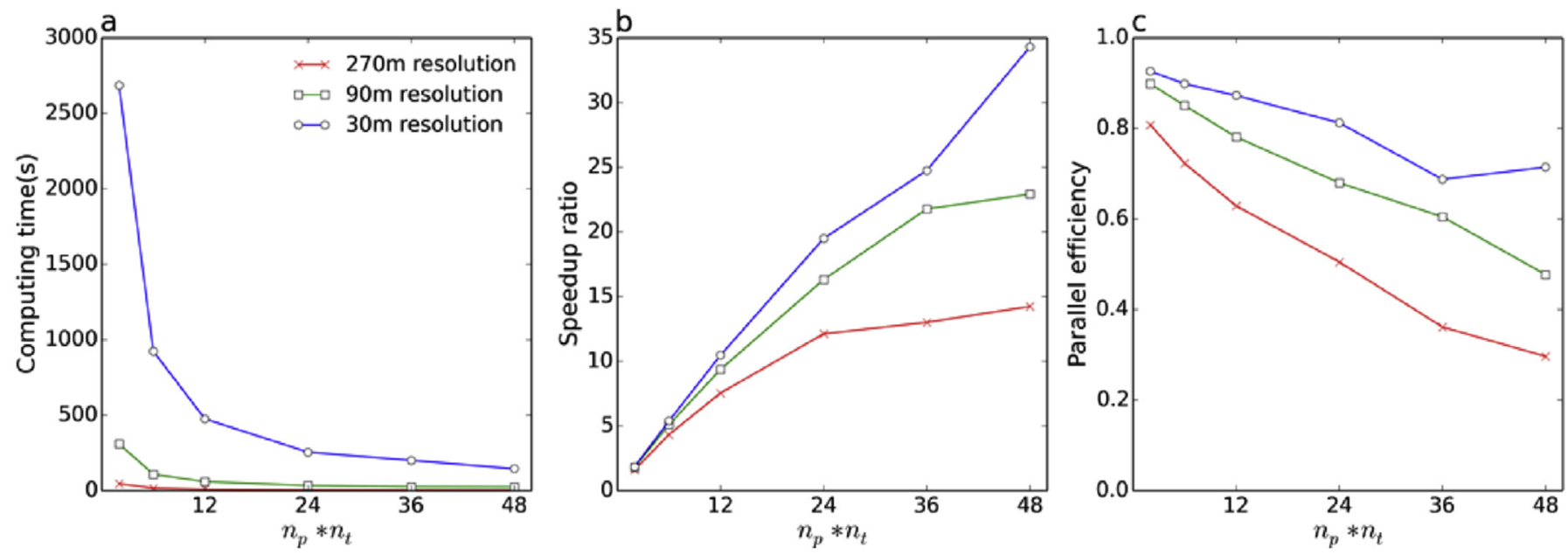

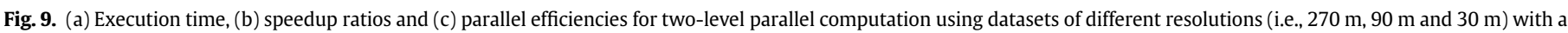
fixed number of sub-basins (i.e., 67). $\mathrm{n}_{\mathrm{p}}$ in this figure means the number of cores and $\mathrm{n}_{\mathrm{t}}$ means the number of threads within each process.

\section{Acknowledgments}

This work was supported by the National Basic Research Program of China (No. 2015CB954102), National Key Technology Innovation Project for Water Pollution Control and Remediation (No. 2013ZX07103006-005), Program of Natural Science Research of Jiangsu (No. BK20150975), National Natural Science Foundation of China (No. 41431177), Program of Natural Science Research of Jiangsu Higher Education Institutions of China (No. 14KJB170009; 14KJA170001) and PAPD (No. 164320H116). Supports to A-Xing Zhu through the Vilas Associate Award, the Hammel Faculty Fellow Award, the Manasse Chair Professorship from the University of Wisconsin-Madison, and the "One-Thousand Talents" Program of China are greatly appreciated. Supports to Junzhi Liu by State Key Laboratory of Resources and Environmental Information System are appreciated.

\section{References}

Band, L.E., Tague, C.L., Brun, S.E., Tenenbaum, D.E., Fernandes, R.A., 2000. Modeling watersheds as spatial object hierarchies: structure and dynamics. Trans. GIS 9 (3), 181-196
Barnett, T.P., Adam, J.C., Lettenmaier, D.P., 2005. Potential impacts of a warming climate on water availability in snow-dominated regions. Nature 438 (17), 303-309.

Bhaduri, B., Minner, M., Tatalovich, S., Harbor, J., 2001. Long-term hydrologic impact of urbanization: a tale of two models. J. Water Resour. Plan. Manag. 127 (1), 13-19.

Borah, D.K., Bera, M., 2004. Watershed-scale hydrologic and nonpoint-source pollution models: review of applications. Trans. ASAE 47 (3), 789-803.

Burger, G., Sitzenfrei, R., Kleidorfer, M., Rauch, W., 2014. Parallel flow routing in SWMM 5. Environ. Model. Softw. 53, 27-34.

Chen, J.M., Chen, X.F., Ju, W., 2013. Effects of vegetation heterogeneity and surface topography on spatial scaling of net primary productivity. Biogeosciences 10 , 4879-4896.

Chow, V., Maidment, D., Mays, L., 1988. Applied Hydrology. McGraw-Hill, New York.

Dehotin, J., Braud, I., 2008. Which spatial discretization for distributed hydrological models? Proposition of a methodology and illustration for medium to largescale catchments. Hydrol. Earth Syst. Sci. Discuss. 12 (3), 769-796.

Garey, M.R., Johnson, D.S., Stockmeyer, L., 1976. Some simplified NP-complete graph problems. Theor. Comput. Sci. 1 (3), 237-267.

Hessel, R., 2005. Effects of grid cell size and time step length on simulation results of the Limburg soil erosion model (LISEM). Hydrol. Process. 19 (15), 3037-3049.

Karypis, G., Kumar, V., 1998. A fast and high quality multilevel scheme for partitioning irregular graphs. SIAM J. Sci. Comput. 20 (1), 359-392.

Kollet, S.J., Maxwell, R.M., 2006. Integrated surface-groundwater flow modeling: a free-surface overland flow boundary condition in a parallel groundwater flow model. Adv. Water Resour. 29 (7), 945-958.

Li, R.M., Simons, D.B., Stevens, M.A., 1975. Nonlinear kinematic wave approximation for water routing. Water Resour. Res. 11 (2), 245-252. 
Li, T.J., Wang, G.Q., Chen, J., Wang, H., 2011. Dynamic parallelization of hydrological model simulations. Environ. Model. Softw. 26 (12), 1736-1746.

Liang, X., Guo, J., Leung, L.R., 2004. Assessment of the effects of spatial resolutions on daily water flux simulations. J. Hydrol. 298 (1), 287-310.

Linsley, R.K., Kohler, M.A., Paulhus, J.L.H., 1975. Hydrology for Engineers. McGrawHIll, New York.

Liu, Y.B., De Smedt, F., 2004. WetSpa Extension, Documentation and User Manual. Department of Hydrology and Hydraulic Engineering, Vrije Universiteit Brussel, Belgium.

Liu, Y.B., De Smedt, F., 2005. Flood modeling for complex terrain using GIS and remote sensed information. Water Resour. Manag. 19 (5), 605-624.

Liu, J.Z., Zhu, A.X., Qin, C.Z., 2013. Estimation of theoretical maximum speedup ratio for parallel computing of grid-based distributed hydrological models. Comput. Geosciences 60, 58-62.

Liu, J.Z., Zhu, A.X., Liu, Y.B., Zhu, T.X., Qin, C.Z., 2014. A layered approach to parallel computing for spatially distributed hydrologic modeling. Environ. Model. Softw. 51 (1), 221-227.

Mattson, T., Sanders, B., Massingill, B., 2004. Patterns for Parallel Programming. Addison-Wesley Professional, Boston.

O'Callaghan, J.F., Mark, D.M., 1984. The extraction of drainage networks from digital elevation data. Comput. Vis. Graph. Image Process. 28 (3), 323-344.

Ran, Q.H., Su, D.Y., Fu, X.D., Wang, G.Q., 2013. A physics-based hydrogeomorphologic simulation utilizing cluster parallel computing. Sci. China Technol. Sci. 56 (8), 1883-1895.

Rauber,, T., Rünger, G., 2010. Parallel Programming: for Multi-core and Cluster Systems. Springer-Verlag New York Inc, New York.

Rojas, R., Velleux, M., Julien, P.Y., Johnson, B.E., 2008. Grid scale effects on watershed soil erosion models. J. Hydrol. Eng. 13 (9), 793-802.
Tripathi, M.P., Raghuwanshi, N.S., Rao, G.P., 2006. Effect of watershed subdivision on simulation of water balance components. Hydrol. Process. 20 (5), 1137-1156.

Vivoni, E.R., Mascaro, G., Mniszewski, S., Fasel, P., Springer, E.P., Ivanov, V.Y. Bras, R.L., 2011. Real-world hydrologic assessment of a fully-distributed hydrological model in a parallel computing environment. J. Hydrol. 409 (1-2) 483-496.

Wang, G.S., Xia, J., Niu, C.W., 2004. Flow routing method and its application in distributed hydrological modeling. Geogr. Res. 23 (2), 175-182 (in Chinese).

Wang, H., Fu, X., Wang, G., Li, T., Gao, J., 2011. A common parallel computing framework for modeling hydrological processes of river basins. Parallel Comput. $37(6-7), 302-315$

Wang, H., Zhou, Y., Fu, X., Gao, J., Wang, G., 2012. Maximum speedup ratio curve (MSC) in parallel computing of the binary-tree-based drainage network. Comput. Geosciences 38 (1), 127-135.

Wang, H., Fu, X.D., Wang, Y.J., et al., 2013. A high-performance temporal-spatial discretization method for the parallel computing of river basins. Comput. Geosciences 58, 62-68.

Yalew, S., van Griensven, A., Ray, N., Kokoszkiewicz, L., Betrie, G.D., 2013. Distributed computation of large scale SWAT models on the Grid. Environ. Model. Softw. 41, 223-230.

Zhao, G., Bryan, B.A., King, D., Luo, Z., Wang, E., Bende-Michl, E., Song, X., Yu, Q. 2013. Large-scale, high-resolution agricultural systems modeling using a hybric approach combining grid computing and parallel processing. Environ. Model. Softw. 41, 98-106.

Zoltay, V.I., Vogel, R.M., Kirshen, P.H., Westphal, K.S., 2010. Integrated watershed management modeling: generic optimization model applied to the Ipswich River Basin. J. Water Resour. Plan. Manag. 136 (5), 566-575. 